notification, and notifying group/casual sex partners to improve partner notification.

\section{HOME SAMPLING THROUGH SOCIAL NETWORK WEBSITES: CAN WE REDUCE UNDIAGNOSED HIV?}

doi:10.1136/sextrans-2012-050601a.20

${ }^{1} \mathrm{E}$ Elliot, ${ }^{*} \mathrm{~A}$ McCowan, ${ }^{1} \mathrm{~S}$ McCormak, ${ }^{1} \mathrm{M}$ Rossi, ${ }^{2} \mathrm{~T}$ Van Every. ${ }^{1}$ Chelsea and Westminster Hospital, London, UK; ${ }^{2} \operatorname{Dr}$ Thom

Background Approximately 1 in 20 men who have sex with men (MSM) in London has undiagnosed HIV. It is a national priority to minimise time spent undiagnosed to reduce morbidity, mortality and onward transmission. Sigma reported that $29 \%$ of MSM recruited online in 2008 had never tested. We piloted an offer of home HIV sampling for MSM in partnership with the Gaydar social network website.

Objectives The objectives of the pilot were to determine (1) uptake of an online HIV risk self-assessment (2) uptake of offer for home testing (3) the proportion of those completing the assessment that is high risk.

Methods Each day 200 MSM in London were invited by Gaydar to assess their HIV risk. "High risk" was defined as unprotected anal sex (UAI) with HIV + partner or of unknown HIV status. Following assessment but regardless of risk, users were offered a postal HIV salivary sampling kit. Reactive results were confirmed in the clinic. Data were analysed using Google Analytics.

Results 4500 invites were issued between 7 November 2011 and 11 January 2012. 363 (8\%) clicked through to the project website, 321 (88\%) of whom completed the HIV risk assessment. Of these, 146 (45\%) were defined as "high risk". 209 clicked through for information on home sampling, 82 (39\%) of whom had never HIV tested. 132 test kits were requested, 73 (55\%) were returned, 4 of which were new HIV diagnoses ( $3 \%$ of kits sent and $5.5 \%$ of samples returned).

Discussion Although low, the uptake appears to reach a population "less tested" than the population that took part in the Sigma online survey or typical of GUM clinics. The level of risk identified was similar to a clinic population with almost half reporting UAI. $55 \%$ of kits were returned and we report $5.5 \%$ or $1: 18$ new diagnoses. The project is now scaling up to message all 120000 Gaydar users in Central London over 12 months and aims to provide evidence to inform the potential roll out of online strategies to enhance community based HIV testing in the UK.
SHOULD WE OPPORTUNISTICALLY TREAT RECTAL CHLAMYDIA TRACHOMATIS INFECTION IN MEN WHO HAVE SEX WITH MEN PRESENTING WITH NON-SPECIFIC URETHRITIS?

doi:10.1136/sextrans-2012-050601a.21

${ }^{1} \mathrm{M}$ Rayment, ${ }^{1} \mathrm{~L}$ Bull, ${ }^{*}{ }^{2} \mathrm{~S}$ Mandalia, ${ }^{1} \mathrm{~F}$ Boag, ${ }^{1} \mathrm{~N}$ Nwokolo, ${ }^{1} \mathrm{R}$ Jones. ${ }^{1}$ Chelsea and Westminster Hospital NHS Foundation Trust, London, UK; ${ }^{2}$ Imperial College

Background Infection of the rectum with Chlamydia trachomatis (CT) is highly prevalent among men who have sex with men (MSM) attending our service (c. 8\%). Our guidelines advise different antibiotics for the treatment of non-specific urethritis (NSU) (azithromycin $1 \mathrm{~g}$ as a single dose) and for rectal CT infection (doxycycline $100 \mathrm{mg}$ twice daily for 7 days). MSM receiving treatment for NSU require recall if they are latterly found to have concurrent rectal CT. Should we therefore use doxycycline first line in MSM presenting with NSU?

Aims To determine the proportion of MSM diagnosed with NSU with rectal CT infection; to identify independent predictors of having rectal CT.

Methods The clinic database identified sequential MSM diagnosed with NSU over 6 months. Co-variables collected: age, sexual history, HIV status, contact history and the presence of rectal symptoms. Only men accepting rectal sampling were included. Univariate and multivariable logistic regression analyses were undertaken.

Results 566 MSM presenting with NSU were identified, of whom $130(23 \%)$ were diagnosed with CT infection of at least one anatomical site. Of this group, 99 were included in the analysis. The overall prevalence of rectal CT infection was 38\% (38/99). In men reporting a history of unprotected receptive anal intercourse (UPRAI) in the preceding 6 months, the prevalence was 61\% (31/ 51); in those without it was 7.1\% (14/48). In MSM reporting rectal symptoms, the prevalence was $90 \%(9 / 10)$; in those without: $33 \%$ (29/89). In the multivariable model, the only independent predictors of risk of rectal CT infection were UPRAI and rectal symptoms (see Abstract O21 table 1).

Discussion First line treatment of NSU in MSM could be modified based on the likelihood of concurrent CT infection. In men reporting UPRAI in the preceding 6 months, or reporting rectal symptoms, it would be reasonable to offer treatment of NSU with doxycycline. In all other patients, we propose continuing to use azithromycin first line, with subsequent recall for further treatment as necessary.

Abstract 021 Table 1 Rate of rectal chlamydia according to history and rectal symptoms

\begin{tabular}{lllllll}
\hline Variable & & Total $(\mathbf{n = 9 9 )}$ & $\begin{array}{l}\text { Number with rectal } \\
\mathbf{C T}(\%)(\mathbf{n = 3 8})\end{array}$ & Adjusted RR & $\mathbf{9 5 \%} \mathbf{~ C l}$ & $\begin{array}{l}\text { Wald statistics, } \\
\mathbf{p ~ V a l u e ~}\end{array}$ \\
\hline $\begin{array}{l}\text { History of unprotected receptive } \\
\text { anal intercourse (UPRAl) }\end{array}$ & $\mathrm{Y}$ & 51 & $31(60.8)$ & 1 & & 0.002 \\
& $\mathrm{~N}$ & 48 & $7(14.6)$ & 0.25 & $(0.07$ to 0.82$)$ \\
Rectal symptoms & $\mathrm{Y}$ & 10 & $9(90)$ & 9.54 & $(1.09$ to 83.4$)$ & 0.041 \\
& $\mathrm{~N}$ & 89 & $29(32.6)$ & 1 & & \\
\hline
\end{tabular}

